TRABAJOS DE PREHISTORIA

51, n. ${ }^{\circ} 1,1994$, pp. $143-156$

\section{ÚTILES DE HUESO \\ DEL NEOLÍTICO FINAL \\ DEL SUR DE LA PENÍNSULA \\ IBÉRICA EMPLEADOS \\ EN ALFARERÍA: \\ PLACAS CURVAS, BISELES, PLACAS Y APUNTADOS}

\author{
SOME BONE TOOLS FROM LATE \\ NEOLITHIC USED IN POTTERY WORK: \\ CURVED PLATES (SLAB), BEVEL-EDGE \\ (CHANFERED EDGE), PLATES AND \\ POINTS (TIPS)
}

\section{MARÍA DOLORES MENESES \\ FERNÁNDEZ (*)}

\section{RESUMEN}

Presentamos el estudio de una serie de objetos de hueso del Neolítico final del Sur de la Península Ibérica, cuyo objetivo es completar la descripción morfométrica con una interpretación tecnológica, a través de la observación traceológica y la experimentación. Junto a éstas, la morfología y metría de cada pieza han sido consideradas en la identificación de sus usos; así, la adaptación de sus formas y dimensiones a determinadas actividades ha contribuido en la tarea de restituirlos al contexto tecnológico del que formaron parte. Se propone también un ejemplo de interdependencia entre el soporte óseo elegido por el artesano, los atributos técnicos de los artefactos manufacturados a partir de dichos soportes y las actividades a los que se destinaron. Todos los objetos proceden de la ocupación neolítica final de un mismo yacimiento, la Cueva de El Toro (provincia de Málaga), y presentan la particularidad común de estar relacionados con el trabajo de la arcilla. La buena conservación de la industria ósea de esta yacimiento ha permitido, pues, identificar algunos componentes del utillaje empleado por los habitantes de esta cueva en una fase concreta de su ocupación.

${ }^{*}$ ) L.A.P.M.O.-Univ. de Provence (U.R.A. 164 du C.N.R.S.). 29, Avenue Robert Schuman. 13621 Aix-en-Provence-Cedex. Francia.

El artículo fue remitido en su versión final el 15-IV-94.

\begin{abstract}
This paper shows the study of a series of bone artefacts from a Late Neolithic site in the south of the Iberian Peninsula. This work intends to complete the morphological and metric descriptions using a technological approach (traceological method and functional perspective). The relationship between this set of bone tools and the pottery works was well-established according to (1st) traceological observation, (2nd) the results from experimental reproduction (manufacture and use principally), and (3rd) the comparison between the morphology of certain pieces (curved plates) and the ceramic one, associated in the same stratigraphic level. The particular features of the bone industry from El Toro Cave permitted us the identification of bone implements pertaining to the kit or case of instruments which prehistoric man needed for the manufacture of ceramic vases.
\end{abstract}

\section{RÉSUMÉ}

Outils en os du Néolithique final du Sud de la Péninsule ibérique employés en poterie: plaques courbes, biseaux, plaques et pointes. Cet article présente l'étude d'une série d'objets en os de morphologie variée datée du Néolithique final du Sud de la Péninsule ibérique. L'analyse de ces outils a été effectuée dans le but de 
compléter la description morphologique et métrique par une interprétation technologique à l'aide d'études tracéologiques et expérimentales. Le but principal fut de déterminer leurs fonctions et de les restituer dans le contexte technologique auquel ils appartinrent. La relation entre cet ensemble d'objets en os et la poterie a été donc établie en fonction de trois procédures : l'observation tracéologique (fabrication mais surtout usage), l'expérimentation développée et la similitude de la morphologie de certaines pièces (plaques courbes) et celle des vases céramiques associés en stratigraphie. Les possibilités offertes par l'industrie osseuse de la Grotte d'El Toro nous ont permit ainsi d'identifier quelques objets appartenant à la trousse d'outils dont eut besoin l'homme préhistorique habitant cette grotte durant le Néolithique final. Nous y proposons aussi un exemple d'interdépendance entre le support osseux choisi par l'homme préhistorique, les traits techniques des artefacts osseux qui en ont été tirés et les usages auxquels ils ont été destinés. En outre, aucune industrie osseuse du Néolithique ou du Chalcolithique en Europe n'ayant permis, à notre connaissance, de retrouver l'ensemble des outils osseux employé en poterie, l'intérêt de la série de pièces que nous présentons ici se trouve renforcé.

Palabras clave: Tecnología ósea. Traceología. Experimentación. Alfarería. Neolítico. Península Ibérica.

Key words: Bone Technology. Traceology. Experimentation. Pottery. Neolithic. Iberian Peninsula.

Mots clé: Industrie osseuse. Tracéologie. Expérimentation. Poterie. Néolithique. Péninsule ibérique.

\section{INTRODUCCIÓN}

En este trabajo se presenta una serie de piezas manufacturadas en hueso, algunas con morfología singular (placas), otras más comunes (biseles o alisadores y apuntados o punzones), procedentes de la Cueva de El Toro (Sierra de El Torcal, Antequera, provincia de Málaga), yacimiento estudiado en el proyecto de investigación dirigido por el Dr. D. Martín Socas (Departamento de Prehistoria, Antropología e Historia Antigua, Universidad de La Laguna) sobre el Neolítico y Edad del Cobre en la comarca de Antequera. La ocupación prehistórica de esta cueva de habitación abarca desde el Neolítico medio hasta la Edad del Bronce (Fig. 1).

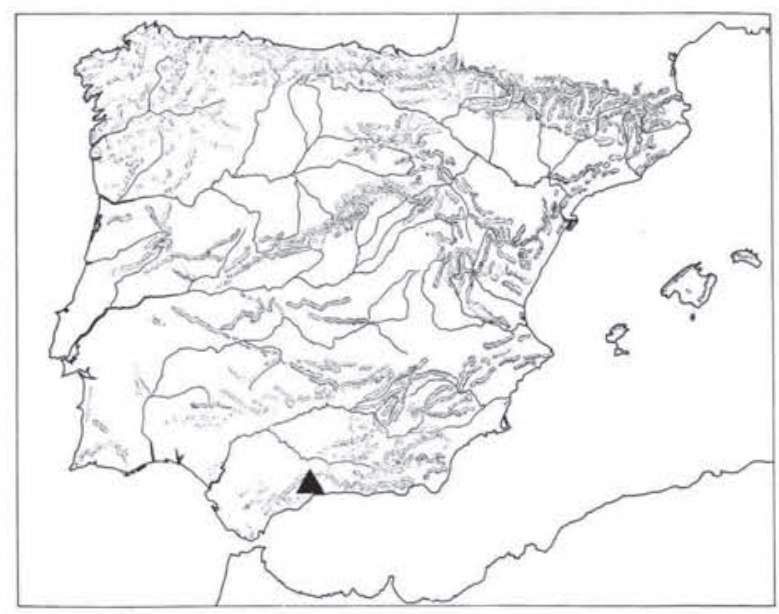

Fig. 1. Localización de la Cueva de El Toro (Sierra de El Torcal, Antequera, Málaga)

El interés de estas páginas es tratar estos artefactos óseos no sólo desde la vertiente tipológica y descriptiva, sino restituyéndolos al conjunto o, si se prefiere, al contexto del que fueron elemento integrante. El estudio de la industria ósea de este yacimiento (Meneses, 1991) ha sido ampliado con una nueva etapa experimental concerniente al uso de ciertos útiles, teniendo en cuenta las fuentes informativas habituales en arqueología experimental: (1) los conocimientos publicados, en este caso no sólo sobre industria ósea sino también en tecnología cerámica prehistórica y etnográfica, (2) los datos derivados de la observación traceológica del material arqueológico y (3) la adecuación de la morfometría de las piezas a unas actividades u otras.

A partir de la observación traceológica del material arqueológico, y del uso sobre varios materiales de nuevas piezas de hueso reproducidas experimentalmente, se percibieron distintas alteraciones en las superficies de los artefactos bien conservados que, una vez comparadas con las huellas de las piezas experimentales, se relacionaron con ciertas actividades; entre ellas el trabajo de la arcilla. La ausencia en la bibliografía de conjuntos óseos elaborados de yacimientos peninsulares con ocupaciones neolíticas y posteriores en los que se haya reconocido los útiles implicados en la industria cerámica, reva- 

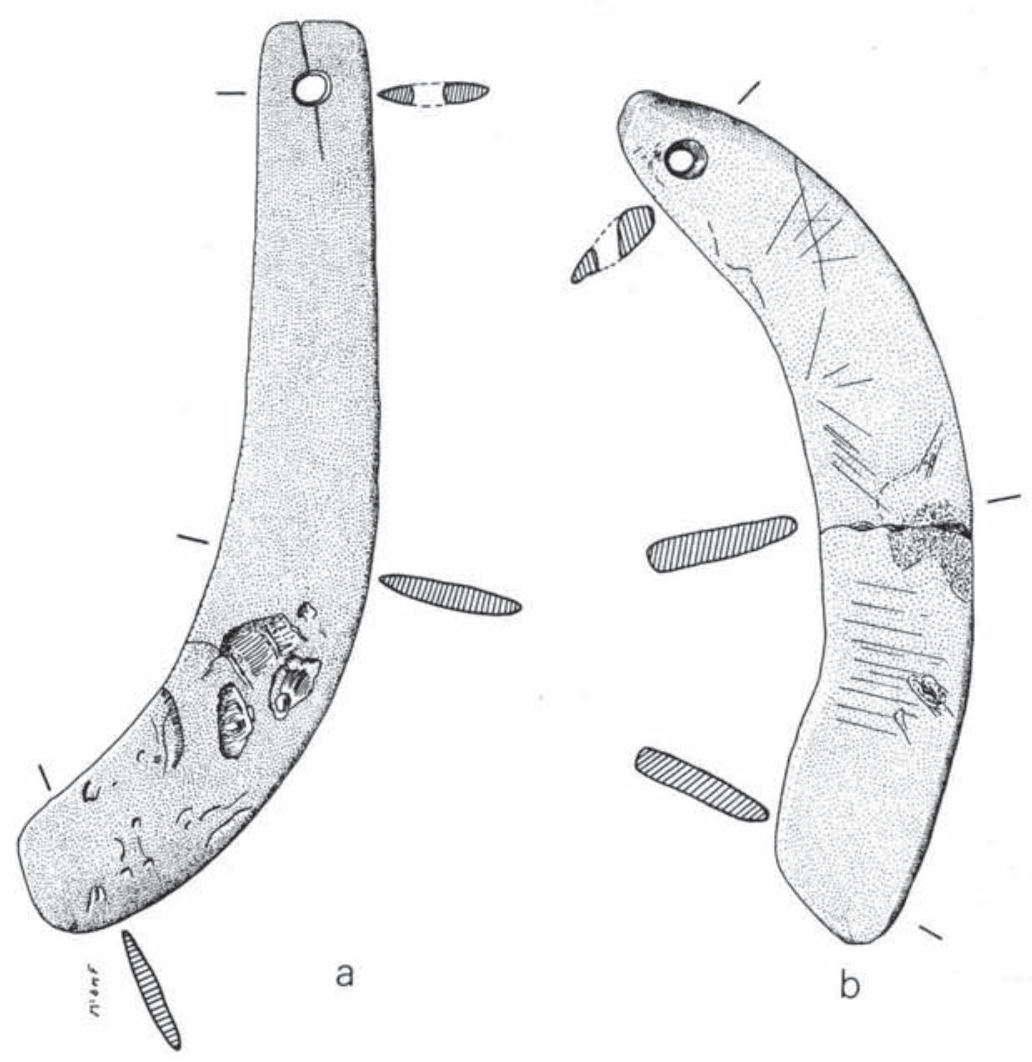

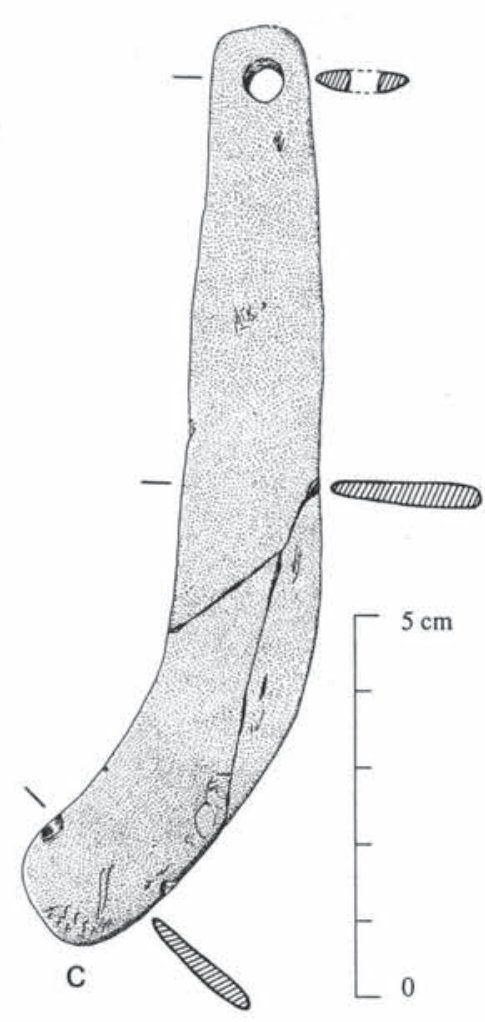

Fig. 2. Placas curvas (a) $\mathrm{n}^{\circ} 1.042 \bullet$ (b) $\mathrm{n}^{\circ} 1.043 \bullet$ (c) 1.044 .

loriza el interés de la serie de piezas presentadas a continuación.

El esquema de este trabajo se articula en torno a las múltiples características de cada objeto: morfología, metría, huellas de fabricación y de uso, y experimentación, sin que ello diluya la relevancia del rasgo común que da unidad a este estudio: la relación útiles óseos-alfarería.

\section{GENERALIDADES}

\section{Definiciones}

A. Placa curva: Objeto de desarrollo longitudinal en curva irregular, con una perforación en uno de sus extremos; realizado sobre hueso largo o plano de rumiante (Fig. 2).

B. Doble-bisel: Objeto alargado con sendos biseles unifaciales en cada extremo; fabricado sobre costilla de rumiante, ambos biseles están localizados en la cara cóncava de ésta (Fig. 3 a).

C. Placa: Objeto alargado con forma irregular y sección de tendencia aplanada, realizado sobre hueso plano, generalmente costilla de rumiante (Figs. 3 b y 4 a).

D. Apuntado: Objeto alargado con un extremo aguzado, realizado sobre metápodo o diáfisis de huesos largos de rumiante (Fig. 4 b y d).

\section{Localización geográfica y cronológica}

A. Las placas curvas sólo han sido halladas hasta el momento en la Cueva de El Toro (1) (Martín et alii., 1987; Martín et alii., en prensa). Los tres ejemplares $\left(\mathrm{n}^{\circ} 1.042,1.043,1.044\right.$; Fig. 2) fueron recuperados en el nivel atribuido a los primeros momentos del Neolítico final (nivel B del estrato III); las dataciones C14 de esta ocupación en El Toro abarcan la segunda mitad del IV milenio a. C.; de la serie de fechas obtenidas, mencionamos las del $3500 \pm 120$ a.C. (GaK$8060)$ y la del $3250 \pm 60$ a. C. (GrN-15437) (Meneses, 1991: 216-217) (2).

(1) Las excavaciones arqueológicas de este yacimiento fueron efectuadas bajo la dirección del Dr. D. Martín Socas.

(2) Los números con los que se presenta cada pieza corresponden a sus números de inventario de excavación. 


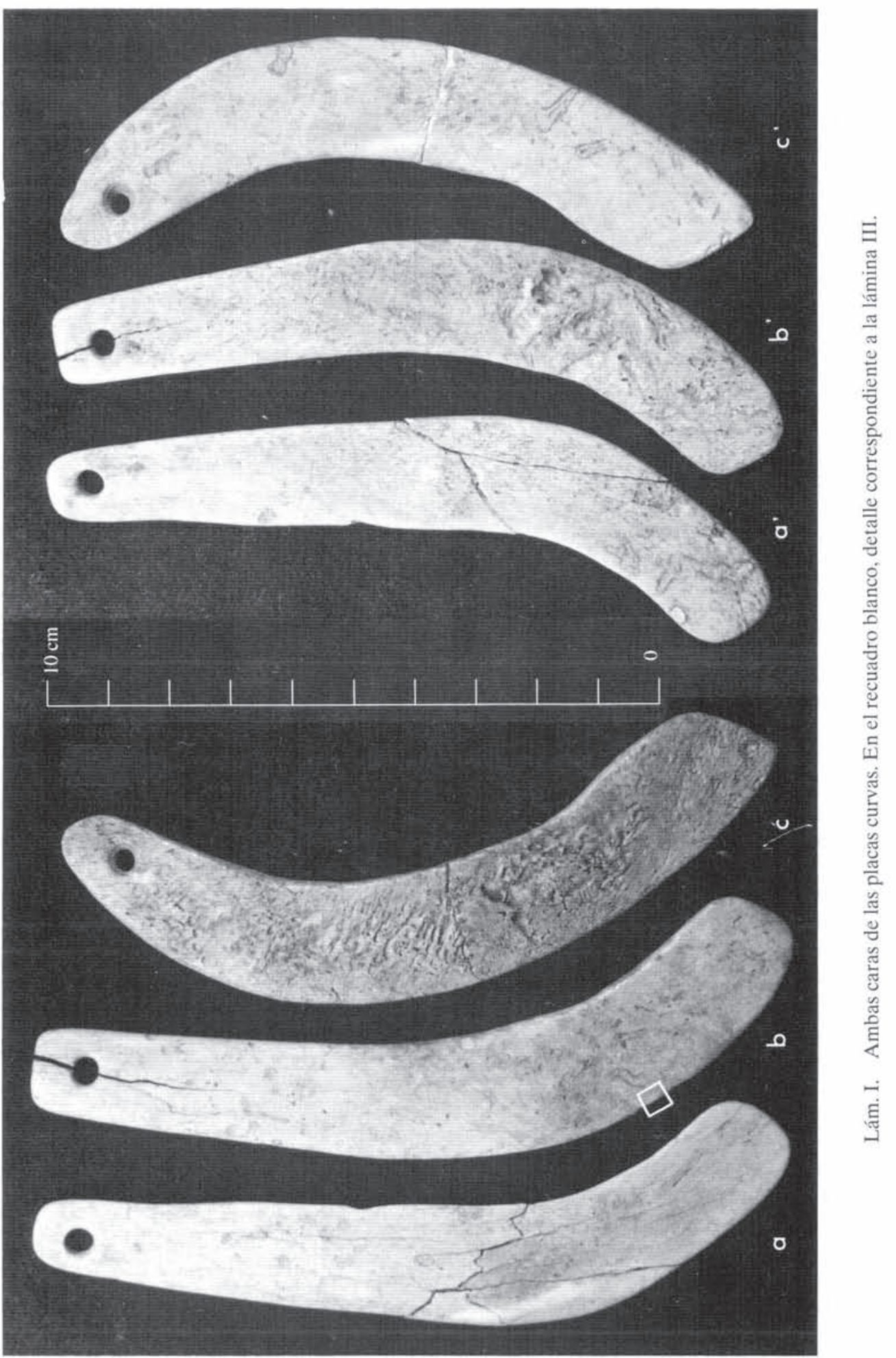

T. P., $51, \mathrm{n}^{\circ} 1,1994$

(c) Consejo Superior de Investigaciones Científicas 
B. Doble-bisel: El único ejemplar $\left(\mathrm{n}^{\circ} 236\right)$ que responde a la definición establecida fue hallado en el nivel superior del estrato del Neolítico final (estrato IIIA, Fig. 3 a).

C. Placas: Pieza $\mathrm{n}^{\circ} 6.050$, Neolítico final (estrato III). Pieza ${ }^{\circ} 37.623$, nivel inferior del Neolítico final (estrato IIIB) (para las dataciones C14 v. supra).

D. Apuntados: De las dos piezas presentadas, la ${ }^{\circ} 1.249$ (Fig. 4 b) procede del estrato correspondiente al Neolítico medio (estrato IV), cuyas cronologías C14 abarcan desde mediados del $\mathrm{V}$ milenio a. C. hasta inicios del IV (v.g. $4450 \pm 150$ a. C. y $4370 \pm 70$ a. C. (Meneses, 1991: 216). La pieza $n^{\circ} 6.832$ (Fig. 4 d) procede del estrato del Neolítico final.

\section{DE LO DESCRIPTIVO: ESTUDIO MORFOMÉTRICO}

\section{A. PLACAS CURVAS \\ Morfología}

La morfología de las tres piezas es la de una placa de sección aplanada y con desarrollo longitudinal en arco de círculo irregular. La similitud de la forma de dos de ellas, las $\mathrm{n}^{\circ} 1.042 \mathrm{y}$ 1.044 (Fig. 2 a y c, Lám. I a-a' y c-c'), con extremos proximales y fustes de tendencia rectilínea, y extremos distales curvos, frente a la pieza ${ }^{\circ}$ 1.043 (Fig. 2 b, Lám. I b-b'), cuyo desarrollo longitudinal describe una curva más bien regular, permitiría establecer dos subtipos. El estado de conservación de los tres ejemplares es completo.

\section{Metría}

- Pieza ${ }^{\circ}$ 1.042: Longitud $118 \mathrm{~mm}$. Grosor mínimo $2 \mathrm{~mm}$.; grosor máximo $3,2 \mathrm{~mm}$. Ancho mínimo 12,6 mm., ancho máximo $20,6 \mathrm{~mm}$.

- Pieza $\mathrm{n}^{\circ}$ 1.043: Longitud $111 \mathrm{~mm}$. Grosor mínimo $1,4 \mathrm{~mm}$.; grosor máximo $5 \mathrm{~mm}$. Ancho mínimo 7,9 mm.; ancho máximo 20,6 mm.

- Pieza n ${ }^{\circ}$ 1.044: Longitud 121,8 mm. Grosor mínimo $1,3 \mathrm{~mm}$.; grosor máximo $3,2 \mathrm{~mm}$. Ancho mínimo 11 mm.; ancho máximo 19,6 mm.

\section{Perforación}

Localizadas en todos los casos en la extremidad proximal, la de la pieza $\mathrm{n}^{\circ} 1.042$ (Fig. 2 a) tiene un diámetro mínimo de $3,6 \mathrm{~mm}$. y máximo de $4,7 \mathrm{~mm}$. El desgaste de su pared impide saber si su realización fue unifacial o bifacial.
El diámetro mínimo de la perforación de la pieza $n^{\circ} 1.043$ (Fig. 2 b) es de 2,9 mm. y el máximo de $5 \mathrm{~mm}$. El desgaste no permite saber si su realización fue unifacial o bifacial.

El diámetro mínimo de la perforación de la pieza $\mathrm{n}^{\circ} 1.044$ (Fig. 1 c) es de $2,8 \mathrm{~mm}$. y el máximo de $4 \mathrm{~mm}$. El escaso desgaste de la pared ha conservado la forma original de su perfil bitroncocónico, atestando su abertura desde ambas caras.

\section{B. DOBLE-BISEL \\ Morfología}

La morfología de ambos extremos, superior e inferior, es en arco de círculo y monobiselada; el fuste corresponde a la diáfisis de la costilla. El estado de conservación es completo.

\section{Metría}

Longitud 156,6 mm. A los $10 \mathrm{~mm}$. del extremo superior, ancho $21,7 \mathrm{~mm}$. y grosor 6,4 $\mathrm{mm}$. Ancho del extremo superior 15,2 mm.; grosor en el mismo punto $1,6 \mathrm{~mm}$. A los $10 \mathrm{~mm}$. del extremo inferior, ancho $16,2 \mathrm{~mm}$. y grosor 4,8 $\mathrm{mm}$. Ancho del extremo inferior $10,8 \mathrm{~mm}$.; grosor en el mismo punto $2 \mathrm{~mm}$. Ancho máximo de la pieza $22,5 \mathrm{~mm}$. Grosor máximo de la pieza $6,6 \mathrm{~mm}$.

\section{PLACAS}

\section{Morfología}

- Pieza n 6.050 (Fig. 3 b): de desarrollo longitudinal ligeramente curvo, la morfología de la extremidad distal es de tendencia curva, monobiselada, de sección plana y con el extremo en arco de círculo. La sección del fuste es también plana, mientras que la del extremo proximal es de tendencia rectangular. Aunque recuperada quemada y fracturada, fue reconstruida completamente.

- Pieza no 37.623 (Fig. 4 a): de desarrollo longitudinal de tendencia rectilínea, la sección a lo largo de toda la pieza es aplanada. Salvo una porción recta del borde izquierdo, el resto de la silueta es irregular. El estado de conservación es completo.

\section{Metría}

- Pieza n ${ }^{\text {: }}$ 6.050: Longitud 117,2 mm. Ancho mínimo $11,3 \mathrm{~mm}$.; ancho máximo $22,6 \mathrm{~mm}$. Grosor mínimo 1,5 mm.; grosor máximo $6,9 \mathrm{~mm}$.

- Pieza ${ }^{\circ}$ 37.623: Longitud mínima 75,4 mm.; longitud máxima 79,5 mm. Ancho mínimo 

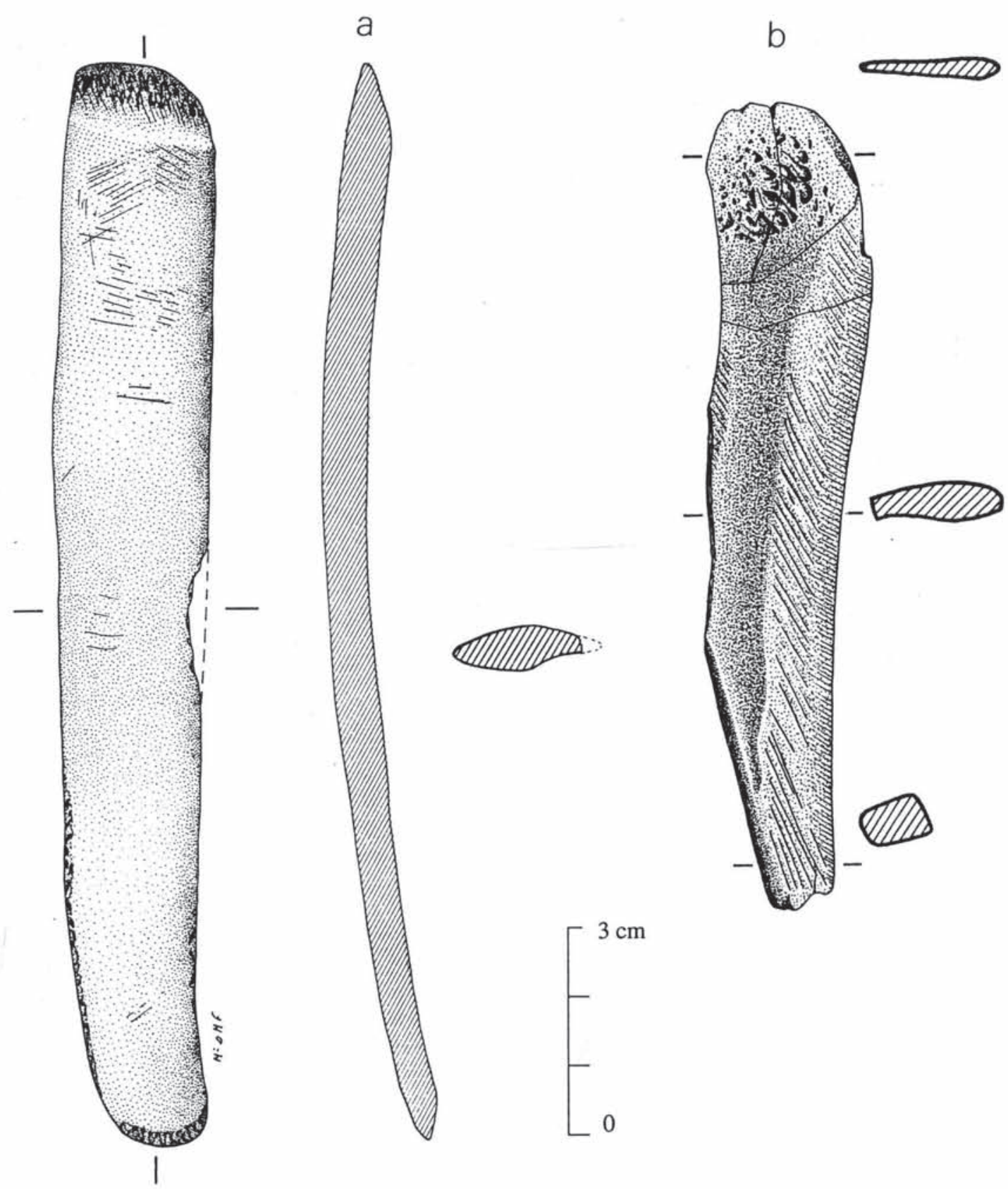

Fig. 3. (a) Doble-bisel sobre costilla de ovis/capra • (b) placa sobre omóplato de bóvido.

T. P., $51, \mathrm{n}^{\circ} 1,1994$

(c) Consejo Superior de Investigaciones Científicas 
21,9 mm.; ancho máximo 26,6 mm. Grosor mínimo $1 \mathrm{~mm}$.; grosor máximo $4,4 \mathrm{~mm}$.

\section{APUNTADOS}

Morfología

Los punzones constituyen uno de los tipos más comunes, sino el más común, de la industria ósea de la prehistoria reciente. Siguiendo la nomenclatura y la tipología establecida por la Commission de Nomenclature sur l'Industrie de l'Os Préhistorique (1990: ficha 8), el apuntado
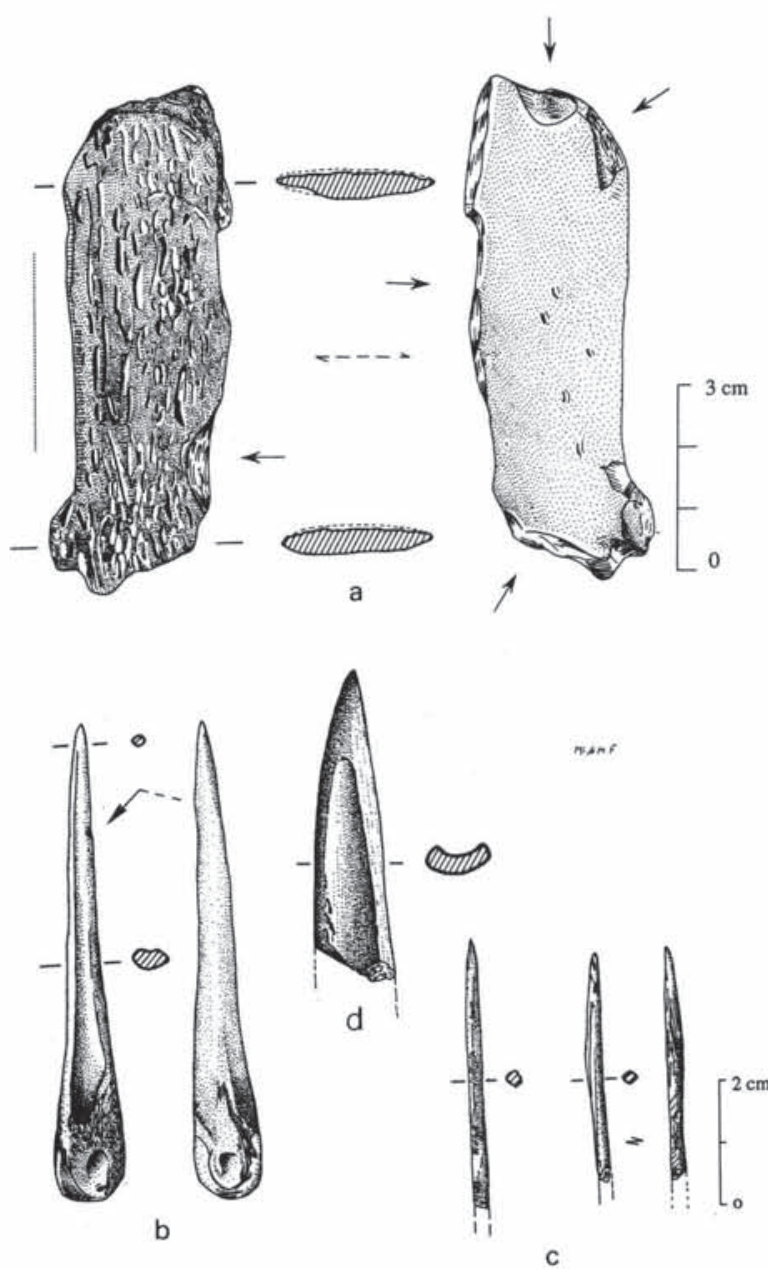

Fig. 4. (a) Placa sobre costilla de ovis/capra. Las flechas indican las esquirlas extraidas por percusión, y la línea de puntos el borde con estrías de uso más marcadas - (b) apuntado sobre metatarso de ovis/capra; la flecha indica sinuosidades típicas de la silueta de una extremidad aguzada por raspado con filo de elemento lítico tallado $\bullet$ (c) astillas óseas similares a las usadas experimentalmente en la decoración de arcilla $\bullet$ (d) extremidad aguzada perteneciente a una pieza empleada en la decoración de cerámica por incisión. $\mathrm{n}^{\circ} 1.249$ (Fig. 4 b) corresponde al tipo denominado poinçon sur métapode fendu de petit ruminant (punzón sobre metápodo de pequeño rumiante hendido longitudinalmente). El estado de conservación es completo.

- El n 6.832 (Fig. 4 d) es un apuntado sobre radio de pequeño rumiante hendido longitudinalmente. Sólo se ha recuperado la extremidad distal y una porción del fuste. Estado de conservación fracturado y quemado.

\section{Metría}

- Pieza n 1.249: Longitud 77 mm. A los 10 $\mathrm{mm}$. del extremo distal, ancho $3,5 \mathrm{~mm}$. y grosor $3,4 \mathrm{~mm}$. A los $10 \mathrm{~mm}$. del extremo proximal, ancho $11,7 \mathrm{~mm}$. y grosor $9,2 \mathrm{~mm}$. Ancho y grosor de la punta $0,6 \mathrm{~mm}$. Extremo proximal, ancho $11,4 \mathrm{~mm}$. y grosor $9 \mathrm{~mm}$.

- Pieza 6.832: Longitud máxima conservada $50,4 \mathrm{~mm}$. Ancho a los $10 \mathrm{~mm}$. del extremo distal $5,6 \mathrm{~mm}$. Ancho máximo conservado $12,7 \mathrm{~mm}$. Grosor máximo conservado 4,3 mm. Ancho y grosor de la punta $0,8 \mathrm{~mm}$.

\section{DE LO DESCRIPTIVO E INTERPRETATIVO: ESTUDIO TÉCNICO Y FUNCIONAL}

\section{A. PLACAS CURVAS}

Materia prima-soporte

Las tres piezas fueron realizadas con gran probabilidad sobre húmeros de mamífero de gran talla (buey o vaca) (Fig. 2, Lám. I). Considerando, de un lado, la conformación de este hueso y, de otro, la identificación en las piezas arqueológicas de cavidades y rugosidades características de la cara interior o medular de los huesos largos, se puede establecer que la porción más idónea para extraer placas con forma curva son, en vista medial o interna, el cuerpo y la extremidad proximal anatómicas.

Técnicas de fabricación y experimentación

A pesar de que las únicas trazas de fabricación conservadas en los tres ejemplares sean estrías finas de pulimento, localizadas en la cara superior y orientadas oblícuamente (NW-SE) (3)

(3) La determinación de la orientación de las estrías de una pieza, respecto a su eje longitudinal o transversal, fue efectuada tras orientar la superficie portadora de cara al observador. El interés de indicar la orientación es doble: precisar la cinemática de la acción y la mano que aprehendía el útil. 


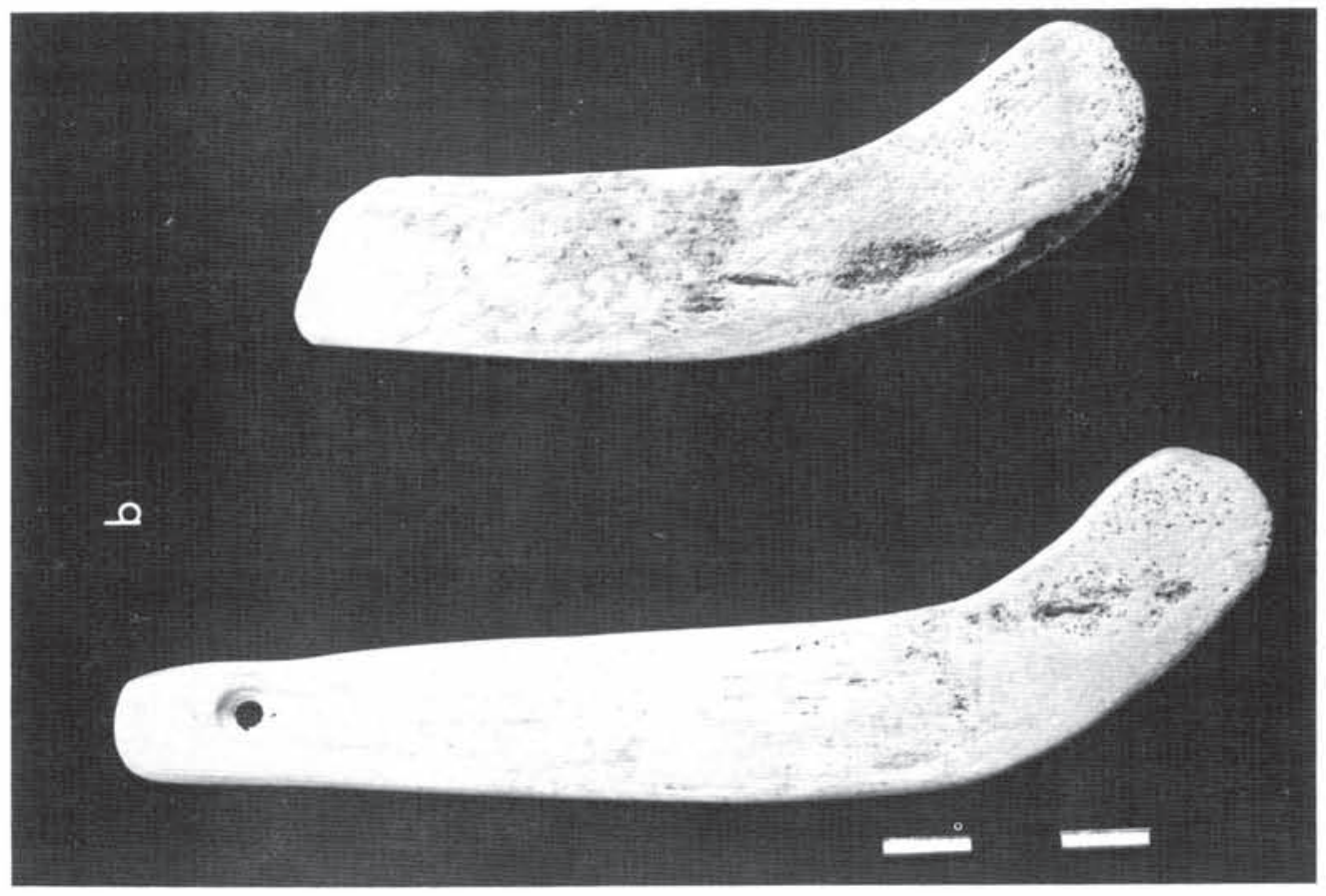


respecto al eje longitudinal de la pieza, las características del soporte (pieza anatómica de gran robustez), el espesor y la forma curva de las placas permiten suponer que la técnica aplicada para su extracción debió de ser el ranurado. Las estrías conservadas corresponderían a la fase final de la fabricación, esto es, al regularizado de las superficies externa e interna originales del hueso, especialmente de las rugosidades del tejido esponjoso.

Las estrías aisladas localizadas en la cara superior, orientadas transversalmente y oblícuamente respecto al eje longitudinal de la pieza ${ }^{\circ}$ 1.043 (Fig. 2, pieza b) son muy probablemente marcas de descarnamiento.

Para la reproducción experimental de piezas similares hemos empleado como soporte omóplatos de carnero y oveja (Lám. II); el trabajo de huesos largos (tibia, fémur, radio y húmero) de buey o de vaca se reveló más dificultoso debido al grosor considerable de las paredes del hueso compacto. En todos los casos el ranurado ha sido la técnica aplicada para la extracción de las placas, grabando primero la silueta de la pieza con la ayuda de un buril, con o sin piqueteado previo, y finalizando el ranurado con láminas y lascas no retocadas.

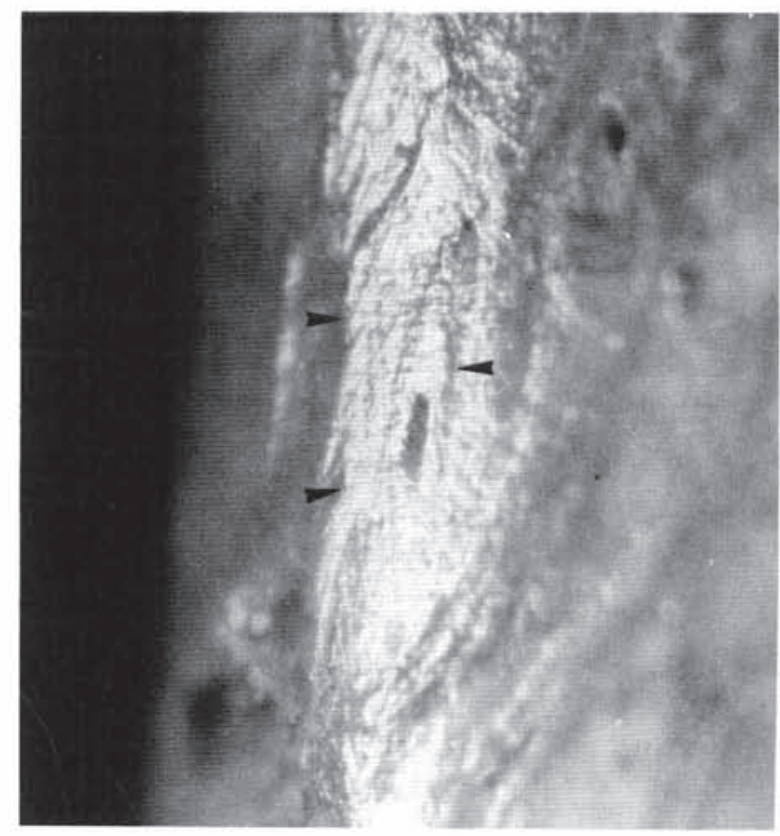

Lám. III. Detalle del borde convexo de la pieza $\mathrm{n}^{\circ} 1.042$; microestrías transversales al eje longitudinal de la placa, paralelas entre sí y asociadas a un lustre intenso de la superficie $(51 \mathrm{x})$.
Los omóplatos permitieron el ranurado desde ambas caras.

\section{Uso y experimentación}

Alteraciones de uso:

Las huellas de uso de las piezas arqueológicas son (1) estrías localizadas en ambos bordes, el convexo y el cóncavo, orientadas trasversalmente al eje longitudinal de la pieza (Lám. III); (2) el desgaste de los bordes convexo y cóncavo, formando un bisel de uso debido al roce

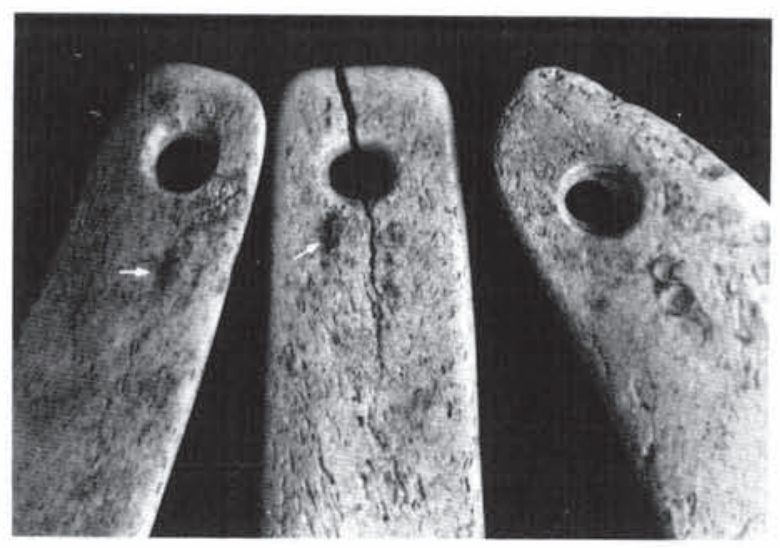

Lám. IV. Detalle de las perforaciones de las placas curvas arqueológicas; las flechas indican restos de arcilla y almagra.

continuado con la materia trabajada; (3) un lustre generalizado a toda la superficie, y (4) restos de almagra y arcilla adheridos a la superficie (Lám. IV).

La perforación en la extremidad proximal facilitaba la sujeción y manejo de la placa en el interior de los recipientes, a la vez que permitía tenerlas atadas, impidiendo su pérdida. La difícil fabricación de tales piezas a partir de huesos largos de mamíferos de gran talla, huesos de considerable grosor a los que se dio una forma nada sencilla de obtener, debió revalorizarlas, tratando su portador de no extraviarlas ni romperlas.

Experimentación:

La utilización de diversas placas, algunas de morfometría similar a las arqueológicas, en el alisado de arcilla ha producido en la superficie estrías finas, un lustre más o menos intenso, dependiendo del grado de depuración de la arcilla, y el redondeado de bordes. 
Las alteraciones de uso descritas, los bordes redondeados por el desgaste, las perforaciones también muy gastadas, la presencia de restos de arcilla y de almagra en la superficie, la coincidencia de los bordes curvos de las placas óseas con la curva de las paredes de ciertos vasos cerámicos del mismo estrato (Figs. 5 y 6), junto al hecho de que las tres piezas aparecieran juntas y asociadas a fragmentos de cerámica en el interior de la cueva, permiten relacionar su uso con la manufactura de recipientes cerámicos. Tales objetos fueron utilizados sobre arcilla a modo de alisador, pero también debieron de utilizarse para dar forma o regularizar las paredes de recipientes cerámicos (4). Las circunstancias del hallazgo de las tres placas óseas no dejan duda sobre su uso simultáneo, quizás por un mismo alfarero.

Se ha comprobado experimentalmente su efectividad en el modelado, regularizado y alisado de paredes y fondo de vasijas, sobre todo de las más abiertas; con el término "modelado" nos referimos a dar a la arcilla la forma del recipiente, y con "regularizado" a sellar, por ejemplo, cordones de arcilla superpuestos en la técnica de urdido.

La dificultad que entraña la fabricación de estas piezas en hueso largos de mamíferos de gran talla no debió favorecer su generalización, al menos en esta materia prima.

\section{B. DOBLE-BISEL}

Materia prima-soporte

El único ejemplar recuperado con la morfología descrita fue fabricado a partir de una costilla de mamífero de talla media, probablemente ovis/capra (Fig. 3 a).

Técnica de fabricación y experimentación

Las huellas identificadas son estrías marcadas ocasionadas por cortes aislados con filo de un elemento lítico tallado, localizadas en la cara cóncava o superior de la pieza y orientadas oblícuamente y transversalmente respecto al eje longitudinal de la misma. El carácter aislado y la disposición irregular de estas estrías hacen pensar más en cortes de descarnización que en alteraciones de fabricación.

(4) Las cerámicas de la Cueva de El Toro han sido estudiadas por el Dr. P. González Quintero (1990) (Departamento de Ciencias Históricas, Universidad de Las Palmas de Gran Canaria, Islas Canarias) en el marco del proyecto de investigación arriba citado.
Nosotros hemos reproducido tres piezas similares en forma y medidas, a partir de costillas de carnero y oveja. Durante el biselado por abrasión de cada extremidad hemos comprobado la mayor facilidad de manipulación del soporte al localizarlos en la cara cóncava. Esta facilidad hace pensar en la elección de la costilla como soporte idóneo para fabricar tal objeto.

\section{Uso y experimentación}

Alteraciones de uso:

Las huellas de uso de la pieza arqueológica consisten en (1) estrías finas localizadas en la superficie biselada de ambos extremos, orientadas oblícuamente (NW-SE) respecto al eje longitudinal de la misma, y (2) en restos de almagra incrustados en el tejido esponjoso.

Experimentación:

El alisado o espatulado de arcilla húmeda produce, además del desgaste de las huellas de fabricación, un lustre acompañado de estrías finas y uniformes ocasionadas por los granos de la pasta. Los extremos biselados de las piezas experimentales tenían una anchura en torno a los $2 \mathrm{~cm}$.; si bien los dos biseles presentes en el ejemplar arqueológico pudieron ser realizados por abrasión, no se descarta un origen funcional; en todo caso, el uso borró las huellas dejadas por la técnica de manufactura.

La reproducción experimental de objetos similares permite comprender el porqué de la localización de los biseles en ambas extremidades de la cara cóncava y no de la convexa: el mejor manejo del soporte, tanto durante la realización de los biseles como de la utilización del objeto sobre las paredes de los recipientes cerámicos; la aprehensión de un objeto empleado en el tratamiento de las paredes de vasos de arcilla es más cómoda si es curvo, adaptándose a la palma de la mano. La orientación de las estrías de las superficies biseladas indica un movimiento de tracción del objeto que monta oblícuamente la pared interna o externa de vaso, en relación al eje vertical de éste (Fig. 6 b). Los doble-biseles son efectivos en el regularizado y alisado de paredes de recipientes de morfología variada.

Lo que podría parecer constricciones técnicas impuestas por el soporte óseo (localización obligada de los biseles en la cara cóncava de la costilla) se revela, tras la experimentación, como una elección más que probable de dicha pieza anatómica por el artesano, tanto por su curvatura natural, adaptable a la palma de la 

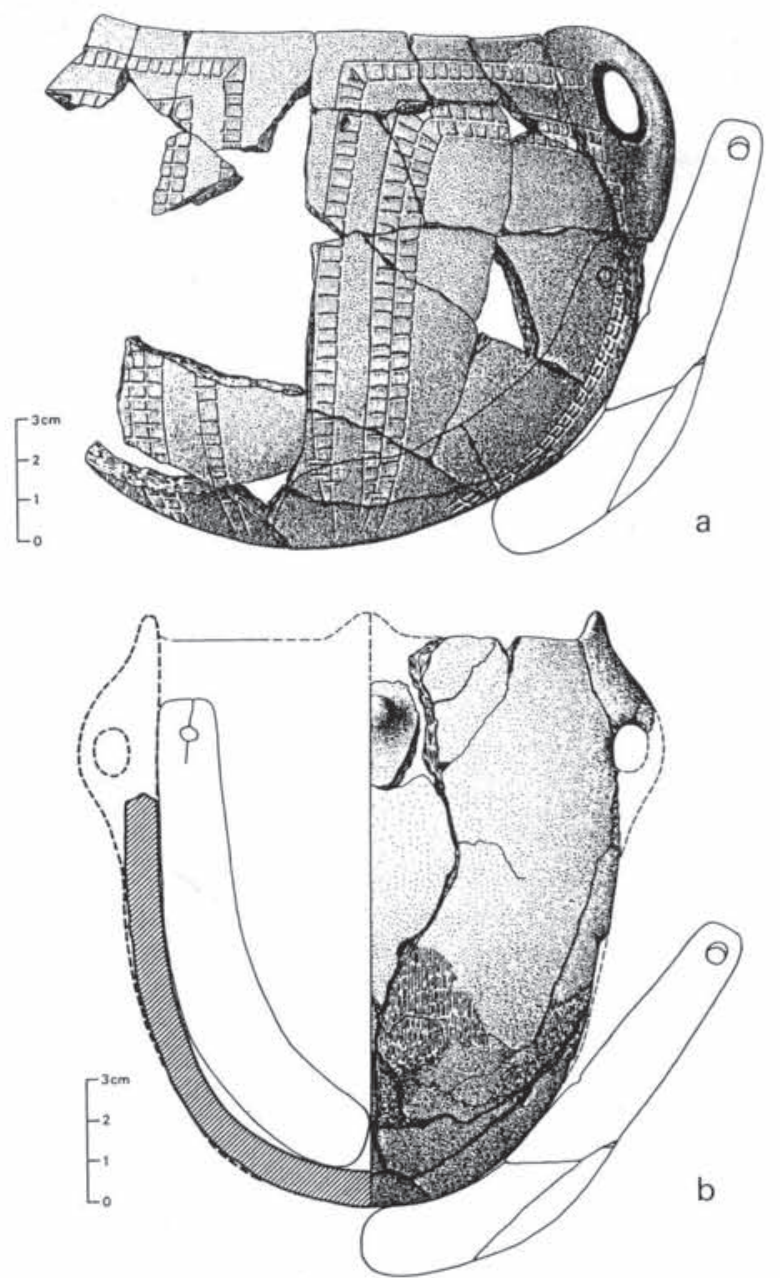

Fig. 5. Montajes propuestos, en un plano, de la utilización de las placas curvas en el alisado de las paredes interiores y exteriores de vasos cerámicos.

mano durante el biselado y la utilización, como por sus dimensiones, permitiendo el acondicionamiento de un frente activo biselado adecuado a la tarea a realizar.

\section{PLACAS}

Materia prima-soporte

- Pieza nº 6.050 (Fig. 3 b): Escápula de mamífero de gran talla, posiblemente de bovino.

- Pieza no 37.623 (Fig. 4 a): Costilla de mamífero de talla media (ovis/capra).

Técnica de fabricación y experimentación

- Pieza n 6.050: Las huellas de fabricación son estrías marcadas ocasionadas por cortes efectuados con filo de elemento lítico tallado; están localizadas en la cara superior y se orien- tan oblícuamenete (NW-SE) respecto al eje longitudinal de la pieza. La cara superior (la dibujada) y el borde izquierdo de la extremidad proximal fueron regularizados por abrasión.

- Pieza n 37.623: Las huellas de fabricación son (1) muescas ocasionadas por episodios de percusión lanzada, localizadas a lo largo del perímetro de los bordes, aplicada para dividir la costilla, y (2) estrías de abrasión para regularizar la cara con tejido esponjoso (Fig. 4 a).

\section{Uso}

- Pieza n ${ }^{\circ}$ 6.050: Clasificada como «paleta», esta pieza presenta como único indicativo de su uso restos de almagra adheridos a su superficie, lo que permite asociarla, al menos, a la manipulación de esta substancia, de gran importancia a lo largo del Neolítico en el Sur de la Península Ibérica ( $c f$. Atoche, 1985, 1986).

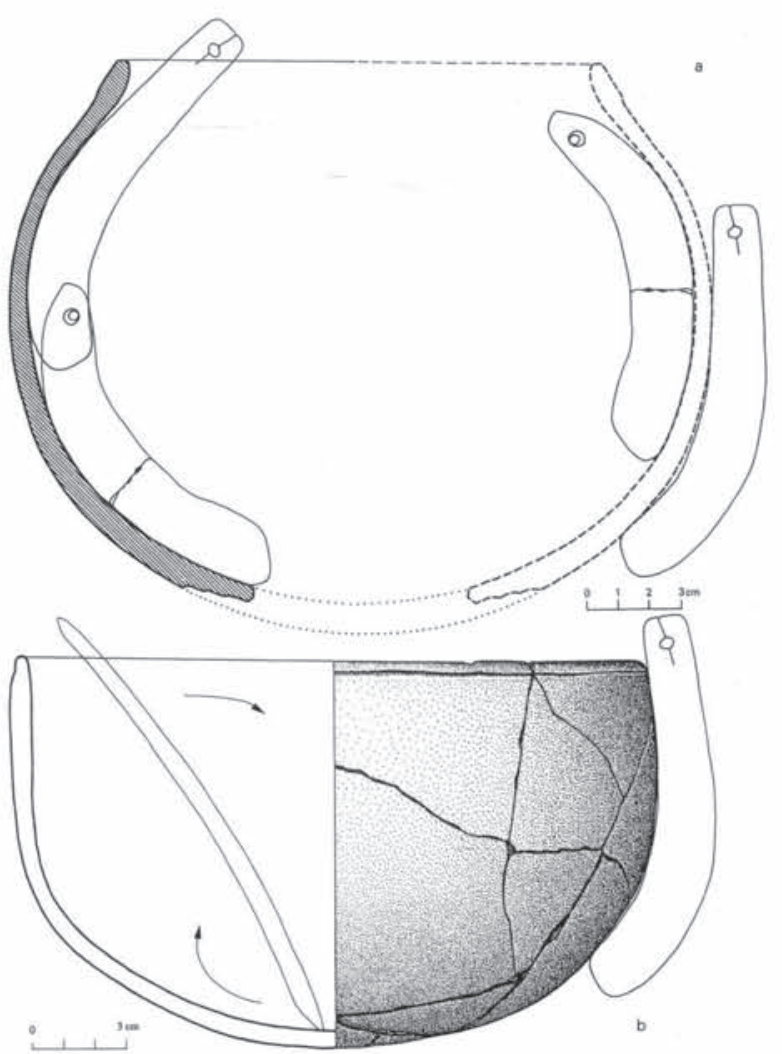

Fig. 6. Montajes propuestos, en un plano, de la utilización de las placas curvas en el alisado de las paredes interiores y exteriores de dos tipos de vasos cerámicos, y movimiento del doble bisel al alisar la pared interna de una vaso cerámico (b). 
- Pieza n ${ }^{\circ}$ 37.623: Las huellas de uso son estrías finas localizadas en los bordes, sobre todo en la porción recta y cara inferior, orientadas transversalmente y oblícuamente (NW-SE) respecto al eje longitudinal de la pieza (borde punteado en la Fig. 3 a). Hay abundantes restos de almagra incrustados en el tejido esponjoso de la cara superior. Las estrías se asemejan a las producidas durante el alisado de arcilla ya descritas al tratarse las placas curvas y el doble-bisel; el movimiento impreso a la pieza fue en dirección $\mathrm{SW}-\mathrm{NE}$ respecto a su eje transversal.

\section{APUNTADOS}

Materia prima-soporte

- Pieza n ${ }^{\circ}$ 1.249: Metatarso de ovis/capra (Fig. 4 b). 4 d).

- Pieza no 6.832: Radio de ovis/capra (Fig.

Técnica de fabricación y experimentación

- Pieza n 1.249: Las huellas de fabricación son (1) estrías ocasionadas por el corte longitudinal del metatarso original con filo de elemento lítico tallado, localizadas a lo largo de ambos bordes, orientadas paralelamente al eje longitudinal de la pieza; (2) estrías de raspado con filo de elemento lítico tallado, localizadas en la cara inferior del fuste y extremo distal, orientadas paralelas al eje longitudinal de la pieza, formando facetas estrechas y largas; (3) ondulación derivada del aguzado por raspado con filo de elemento lítico tallado, localizada en el borde derecho del fuste distal (flecha en la Fig. 4 b); (4) estrías de pulimento, localizadas en el extremo proximal y orientadas oblícuamente (NW-SE) respecto al eje longitudinal de la pieza, y (5) "chattemarks" (5) localizadas en la extremidad distal y orientadas transversalmente respecto al eje longitudinal de la pieza.

- Pieza no: 6.832: Las huellas de fabricación son (1) estrías ocasionadas por el corte longitudinal del radio original con filo de elemento lítico tallado, localizadas a lo largo de ambos bordes y orientadas paralelamente al eje longitudinal de la pieza; (2) estrías de raspado con filo de elemento lítico tallado, localizadas en el

(5) Se entiende por "chattemarks» en traceología ósea las suaves marcas transversales a la dirección de la acción, ocasionadas por pequeños y contínuos rebotes del filo lítico tallado sobre el hueso raspado; rebotes a su vez debidos a la resistencia que opone la materia ósea trabajada al filo. Son evidencia de la aplicación de la técnica del raspado. borde derecho de la extremidad distal y en ambas caras, orientadas paralelamente al eje longitudinal de la pieza, y (3) estrías de pulimento localizadas en la cara superior y bordes de la extremidad distal, orientadas oblicuamente (SW-NE) respecto al eje longitudinal.

Uso y experimentación

Alteraciones de uso:

- Pieza no 1.249: Las huellas de uso son (1) el desgaste de las estrías de aguzado de la extremidad distal, (2) lustre en la superficie de la extremidad distal, (3) muesca de astillado de la punta, (4) estrías finas localizadas en la extremidad distal orientadas oblícuamente respecto al eje longitudinal de la pieza y (5) restos de almagra en la superficie.

- Pieza $n^{\circ}$ 6.832: Las huellas de uso son (1) el desgaste de las estrías de aguzado de la extremidad distal, (2) estrías finas y cortas, localizadas en la extremidad distal y orientadas oblícuamente y transversalmente respecto al eje longitudinal de la pieza y (3) desgaste asimétrico de la punta hasta volverla roma.

Experimentación:

Para la decoración de arcilla utilizamos astillas de hueso, productos de desecho de la fabricación de otros objetos; la morfología de éstas era similar a la de las piezas arqueológicas representadas en la figura $4 \mathrm{c}$. Las alteraciones producidas en la superficie del extremo activo, tras incidir repetidas veces la arcilla húmeda, fueron (1) finísimas estrías delimitadas al extremo, (2) el desgaste asimétrico de éste y (3) un lustre asociado.

Tales alteraciones de uso descartan el empleo de la pieza $n^{\circ} 1.249$ en la perforación, por ejemplo, de pieles, dado que esta acción produce huellas diferentes en la superficie de la extremidad activa. Según el aspecto de la superficie de esta extremidad y la forma roma adoptada por su borde distal, este apuntado debió de utilizarse en la decoración de cerámicas. El desgaste asimétrico de la silueta de la punta y la formación de estrías no abarcan más de 2 ó $3 \mathrm{~mm}$. desde el borde distal hacia el fuste; la posición inclinada sobre la pasta es lo que produce el embotado asimétrico de la punta. 


\section{DISCUSIÓN Y CONCLUSIONES}

Las informaciones etnográficas constituyen una fuente documental aprovechada también en el estudio de la industria ósea prehistórica, especialmente en lo que concierne al reconocimiento del uso de los útiles por comparación. Considerando las analogías etnográficas en su justo valor -el de una fuente sugerente de hipótesis, y no como medio de validar la reconstrucción funcional propuesta aquí-, creemos oportuno añadir este comentario en el que se resumen algunos datos etnográficos a estimar.

Las piezas que quizás atraen más la atención por su excepcional morfología son las placas curvas. Se ha ampliado la búsqueda de símiles prehistóricos y etnográficos más allá del espacio geográfico y cronológico en el que se inscribe la Cueva de El Toro. Para los primeros, el rastreo bibliográfico no ha aportado yacimiento alguno de la prehistoria reciente de la Península Ibérica, ni de Europa en general, con piezas similares. Para los segundos, la consulta de trabajos etnográficos sobre la tecnología cerámica de comunidades de ubicaciones geográfica y cronológica diversas ha confirmado la utilidad potencial de piezas con tal morfología, cuyo empleo se asemejaría al alisado de la pared interna de recipientes cerámicos con la ayuda del dedo índice flexionado (Fayole, 1992: 71-72, 75-77, figs. $29 \mathrm{n}^{\circ} 1$ y $\left.30 \mathrm{n}^{\circ} 4\right)$.

La mayoría de los trabajos editados sobre tecnología cerámica se centran en la "cerámica objeto acabado", obviando habitualmente los útiles implicados en su manufactura. Esta limitación no ha facilitado documentar piezas asimilables morfométricamente a las arqueológicas presentadas aquí entre los útiles de hueso, madera u otra materia, empleados en la manufactura de cerámicas en diversas comunidades prehistóricas y actuales (cf. Gibson y Woods, 1990; Longacre, 1991). Cuando se alude a los objetos empleados en la decoración de cerámica, los autores se ciñen a lo morfológico, no interesándose por sus características traceológicas.

Concluyendo, este estudio quiere constituir un ejemplo de la interdependencia entre la elección de los soportes óseos, los rasgos técnicos de los artefactos óseos en que derivaron y los usos a los que los destinó el hombre prehistórico. Las condiciones bien documentadas del hallazgo de los objetos presentados, la ade- cuada conservación para su análisis traceológico y el conocimiento del material no óseo asociado en estratigrafía, en este caso el cerámico, han facilitado completar la fase descriptiva morfométrica. Pero si bien la traceología no ha permitido la reconstrucción técnica total de todas las piezas (recordemos que la transformación intensa de las placas curvas borraron las alteraciones debidas a su fabricación, también irreconocibles en el bisel), las alteraciones de uso identificadas en las superficies de todas las piezas, la presencia de restos de almagra y arcilla adheridos a la superficie de alguna de ellas, la pertinencia de los resultados de la experimentación efectuada con las piezas reproducidas y, por último y en lo que concierne a las placas curvas, la morfología de los vasos cerámicos asociados en estratigrafía, parecen ser factores concluyentes a la hora de vincular esta serie de artefactos óseos a la manufactura de recipientes cerámicos.

\section{Agradecimiento}

Al Museo Arqueológico y Etnográfico de Tenerife y al Departamento de Prehistoria, Antropología e Historia Antigua de la Universidad de La Laguna (Tenerife).

\section{BIBLIOGRAFÍA}

Atoche Peña, Pablo (1985): «La cerámica a la almagra en las Cuevas de Andalucía Oriental». Anuario 82-83, Universidad de La Laguna. Tomo I-Libro $1^{\circ}$, Derecho, Económicas y Geografía e Historia: 49-64. La Laguna.

- (1986): «La cerámica a la almagra en las cuevas de Andalucía Oriental. Su encuadre en el Neolítico de Granada y Málaga». Memoria de Tesis Doctoral. Universidad de La Laguna (inédita).

COMMISSION DE NOMENCLATURE SUR L'INDUSTRIE DE L'OS PRÉHISTORIQUE (1990): Cahier III «Poinçons, Pointes, Poignards, Aiguilles». Fiches Typologiques de l'Industrie Osseuse Préhistorique (H. Camps-Fabrer respons). Publications de l'Université de Provence. Aix-en-Provence.

FAYOLE, Véronique (1992): La poterie modelée du Maghreb oriental. De ses origines au XXe siècle. Technologie, morphologie, fonction. Ed. du C.N.R.S. Centre Régional de Publications de Marseille. Paris, 298 pp., 101 figs., 22 láms. 
Gibson, Alex \& Woods, Ann (1990): "Prehistoric Pottery for the Archaeologist". Leicester University Press. Leicester.

González Quintero, Pedro (1990): La Cerámica de la Cueva del Toro y la Secuencia del Neolítico Malagueño. Serie Tesis Doctorales. Secretariado de Publicaciones de la Universidad de La Laguna. La Laguna (Microfichas).

LONGACRE, William A. (ed.) (1991): "Ceramic Ethnoarchaeology". The University of Arizona Press. Tucson.

Martín Socas, D.; Camalich Massieu, M. ${ }^{a}$ D. y GONZÁLEZ QUINTERO, P. (1987): «Informe preliminar de la campaña de 1985 en la Cueva de El Toro de El Torcal (Antequera, Málaga)». Anuario Arqueológico de Andalucía 1985, II, 233-240. Sevilla.
Martín, D.; Camalich, M. D. D.; Buxó, R.; GonZÁlez, P.; Hernández, J. M.; Martínez, G.; Mederos, A.; MeNeses, M. ${ }^{a}$ D.; De PAZ, M. A.; Risco, F. y WATSON, J. (en prensa): «Resultados de los trabajos de excavación en la Cueva de El Toro (El Torcal, Antequera, Málaga)». Campaña de 1988. Anuario Arqueológico de Andalucía 1988, II. Sevilla.

Meneses Fernández, María Dolores (1991): Propuesta Metodológica para el Análisis de la Industria Ósea del Neolítico en Andalucía. Un Ejemplo: la Cueva del Toro (Antequera Málaga). Serie Tesis Doctorales. Secretariado de Publicaciones de la Universidad de La Laguna. La Laguna, 536 pp., 53 figs., 49 láms. (Microfichas). 